\title{
Evaluation of Biologically Active Compounds from Calendula officinalis Flowers using Spectrophotometry
}

\author{
Monica Butnariu $^{1 *+}$ and Cristina Zepa Coradini ${ }^{2}$
}

\begin{abstract}
Background: This study aimed to quantify the active biological compounds in C. officinalis flowers. Based on the active principles and biological properties of marigolds flowers reported in the literature, we sought to obtain and characterize the molecular composition of extracts prepared using different solvents. The antioxidant capacities of extracts were assessed by using spectrophotometry to measure both absorbance of the colorimetric free radical scavenger 2,2-diphenyl-1-picrylhydrazyl (DPPH) as well as the total antioxidant potential, using the ferric reducing power (FRAP) assay.

Results: Spectrophotometric assays in the ultraviolet-visible (UV-VIS) region enabled identification and characterization of the full range of phenolic and flavonoids acids, and high-performance liquid chromatography (HPLC) was used to identify and quantify phenolic compounds (depending on the method of extraction). Methanol ensured more efficient extraction of flavonoids than the other solvents tested. Antioxidant activity in methanolic extracts was correlated with the polyphenol content.

Conclusions: The UV-VIS spectra of assimilator pigments (e.g. chlorophylls), polyphenols and flavonoids extracted from the $C$. officinalis flowers consisted in quantitative evaluation of compounds which absorb to wavelengths broader than $360 \mathrm{~nm}$.
\end{abstract}

\section{Background}

Owing to their distinct composition and economic value, cultivation of marigolds (C. officinalis) continues to increase [1], and the European Union has sponsored several programs for the investigation and exploitation of this species [2-4]. The therapeutic properties of marigolds are attributed to the diverse range of biologically active substances they contain [5]. Owing to their antiinflammatory, healing, and antiseptic properties, C. officinalis extracts are applied externally to treat various skin ulcerations, eczema and conjunctivitis, and internally to soothe pain arising from stomach ulcers and inflammation. Marigold is renowned for its antispasmodic, aperient, cholagogic, diaphoretic and vulnerary

\footnotetext{
* Correspondence: monicabutnariu@yahoo.com

† Contributed equally

${ }^{1}$ Chemistry and Biochemistry Department,Banat's University of Agricultural Sciences and Veterinary Medicine from Timisoara, Calea Aradului, no. 119, Timisoara, 300645 Romania

Full list of author information is available at the end of the article
}

properties [6]. Phenolic compounds (flavonoids and phenolic acids) and saponins are both abundant in marigold [7].

C. officinalis also contains carotenoids and triterpenic alcohols, both in their free and esterified forms $[8,9]$. $C$. officinalis-derived carotenoid pigments [10] and polyunsaturated fatty acids, such as calendic acid [5], have been shown to have inflammatory properties in vivo and inhibitory properties in vitro [11].

Marigolds are also characterized by the presence of some important polycarbohydrates [12], which are soluble in water, and play a role in tissue soldering and controlling cellular permeability [13-15]. Other substances identified in C. officinalis extracts are proteins and amino acids [16], saturated hydrocarbons [17], vitamin $C$ [18] and mineral substances [19]. Given their strong aromas [20], etheric oils could attract insects, thereby promoting pollination indirectly [21-23].

Some volatile substances [24] represent final products of anaerobic biodegradation of carbohydrates [25] 
accumulate to levels comparable to those synthesised in cells [26].

Besides their role as antioxidants [27], polyphenols (especially flavonoids) have antimicrobial properties $[28,29]$.

Our previous studies used chromatography to demonstrate that marigold is a good source of natural antioxidants, in particular polyphenolic substances.

Polyphenols are normally characterized by their specific chromatographic behaviours and distinctive UV-VIS spectra.

The objective of this study was to use spectrophotometry to evaluate the contents of phenolic and flavonoid compounds, and the radical-scavenging activity in extracts of different marigold samples. The main phenolic compounds in C. officinalis tested in this study were systematically identified by comparing HPLC profiles of samples with those of authentic phenolic standards and published data (information in patents RO122836-B1) [30]. We used HPLC to simultaneously separate, detect and quantify phenolic compounds, chlorophylls and carotenoids in a single run. The availability of a method to obtain a bioactive profile of different vegetation matrices should help to meet increasing demands for information on biological compounds and their properties, not only in marigold flowers but also in other natural food sources.

\section{Results}

\section{Spectrophotometric assays}

The present research describes a Folin-Ciocalteu (FC) method for the analysis and the quantification in fast time. The fast method was validated in term of repeatability, reproducibility, linearity, accuracy, optimised analytical method were then conducted in order to quantify phenolic compounds. Spectrophotometric assays to determine the total flavonoid and polyphenol contents of plant extracts were adapted for use with a UV-VIS spectrophotometer (PG Instruments) and soft UV WIN 5.05 to measure the absorbances of reference solutions (QE and GA), and the absorbances of samples from plant extracts [31]. Spectrophotometric assays for the calculation of concentrations of quercetin (QE) were based on absorbance measurements taken at $420 \mathrm{~nm}$. Spectrophotometric determination of gallic acid (GA) content was calculated at $765 \mathrm{~nm}$. The linearity of the method was verified through the method of least squares, which was applied in the concentration range 2-32 $\mu \mathrm{g} / \mathrm{mL}$ for $\mathrm{QE}$ and $1-10 \mu \mathrm{g} / \mathrm{mL}$ for GA, with a correlation coefficient $R^{2}>0.99$. To verify the results, replicate calibration curves were compared to the reference substances for three consecutive days. A slight difference was observed between the calibrations curves generated for QE, whereas those for GA were nearly identical. All the determination factors of equations of calibration straight lines $\left(R^{2}\right)$ were greater than 0.99 . The precision of the method was calculated by comparing the determined quantity of the substance with the quantity of substance calculated based on statistical analysis using the program ORIGIN 7 (Standard/Professional versions). For all control samples, differences between experimental samples and standards were never statistically significant $(P<0.05)$. The accuracy (percentage deviation of the concentration determined relative to the calculated concentration) and the precision (standard deviation/ coefficient of variation) of methods always fell within accepted ranges $( \pm 15 \%$ of the mean).

The sensitivity of the method was calculated using 2 $\mu \mathrm{g} / \mathrm{mL}$ of $\mathrm{QE}$ and $1 \mu \mathrm{g} / \mathrm{mL}$ of GA. In both instances, when the accuracy and precision framed in admitted limits in such determinations added up to the quantification limit $( \pm 20 \%)$.

Phytochemicals and in particular phenolic acids and flavonoids compounds present in C. officinalis flowers may be partially responsible for these health benefits through their antioxidants activity. In the present study, the main goals consist in isolating total flavonoid and polyphenolic compounds by spectrophotometric assays in the ultraviolet-visible. To distinguish rapidly between various samples, flavonoids were extracted from flowers by immersing (without crushing the tissue) in solvents (HPLC method, we investigation for a patent RO122836-B1).

\section{High Performance Liquid Chromatography}

In accordance with the international standards of the Organic Crop Improvement Association (OCIA), the International Federation of Organic Agriculture Movements (IFOAM) and the National Organic Program of the U.S. Department of Agriculture, the use of environmentally friendly extraction procedures, such as maceration, is needed to obtain such natural ingredients.

We used an objective HPLC assay coupled to spectrophotometric methods for exhaustive characterization of polyphenolics, including compounds with antioxidant potential such as flavanols, phenolic acids and carotenoids, from an extract of $C$. officinalis flowers. The most abundant phenolics were quantified using HPLC by comparison with external standards of known phenolics.

\section{Total polyphenol, flavonoid content and estimation of antioxidant activity using DPPH and FRAP}

Concentrations of polyphenols in experimental samples varied according to the type of plant material and the solvent used for extraction. Values determined using the DPPH method (Additional File 1 and Figure 1) showed that antioxidant activity was significantly greater with the variety $P_{2}$ than the variety $P_{1}$. This might be 
(a) Total flavonoid content and total polyphenolic content

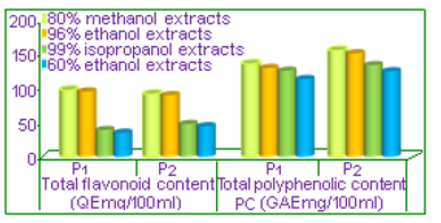

(b) Ferric reducing antioxidant power and DPPH radical scavenging activity.

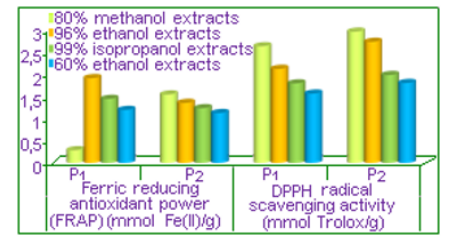

Figure 1 Contents of compounds with antioxidant potential from $C$. officinalis extracts.

explained by greater enzymatic activity in the former. Measurements were taken between the wavelengths 440 and $750 \mathrm{~nm}$. All scans obtained using experimental samples were compared with those obtained using pure solvents. Measurements were taken $30 \mathrm{~min}$ after addition of DPPH in order to ensure enough time for the antioxidants to react with DPPH. As shown in Additional File 1 absorbance changes at this wavelength indicate whether DPPH radicals are in an oxidized or reduced state. Thus, this wavelength enables a straightforward analysis and interpretation of the measured decrease in absorbance. The hydrophilic antioxidant activity was evaluated using both the FRAP and DPPH methods. Antioxidant activity was greater in samples from the variety $\mathrm{P}_{2}$ samples than those from the variety $\mathrm{P}_{1}$, possibly owing to greater levels of biosynthesis in the former than in the latter. The use of FRAP methods resulted in similarly small values for all three types of extracts. The huge distribution of materials and biological liquids of the biologically active molecule did not facilitate the adoption of a general method-available for extractionwhich could be applied in a general way, and adopted as standardized technic. The wide range of analytes and solvents used prevented us from using a standard extraction procedure throughout the study. Extraction of carotenoids using the unipolar organic solvents [32] ethylic ether or chloroform involved maceration of plant tissue followed by mechanical agitation and transfer of the extracts to a boiling water bath. A considerable amount of trituration was necessary to ensure complete extraction; because the unipolar solvent did not penetrate the cellular membrane to dissolve the carotenoids. As fresh material has high levels it must first be dried prior to extraction with miscible solvents, such as acetone, methanol, and ethanol $[33,34]$. The plant material might then be sufficiently anhydrous to enable extraction using non-aqueous solvents.

The objective of this experimental part was to the quantification of antioxidant activity by spectrophotometry (Additional File 1). We developed a simple and efficient method for extracting. It was to identify flavonoids in its extracts, to determine the quantity and make a projection on its antioxidant capacity by DPPH spectrophotometric method. The model of scavenging the stable DPPH radical is a widely used method to evaluate the free radical scavenging ability of various samples.

\section{Pigment Analysis}

Organic extracts contained carotenoids as well as some waste substances, such as chlorophyll $[35,36]$. Analysis of the results from Additional File 2 reveals differences in the contents of assimilator pigments in the analysed samples. The higher quantities of chlorophyll $b$ (Figure 2 and Figure 3) suggest the capacity of flower tissues to capture light radiation to enhance the capacities of the plants to convert light energy into potential chemical energy. Samples that produce larger quantities of carbohydrates are derived from tissues that provide substances needed for development [37].

As previously reported [38], a quantasome (complex between photosynthetic pigments and protein-phosphorus membranes of grana) [39] comprises 230 chlorophyll (Chl) molecules $(160 \mathrm{Chl} a$ and $70 \mathrm{Chl} b$ molecules) and approximately 50 carotenoid molecules. Chlorophyll $a$ molecules act as reaction (trap) centres ( $\mathrm{P}_{700}$ in photosystem (PS) I and $\mathrm{P}_{680}$ in PS II). Theoretically, the Chl $a$ : Chl $b$ ratio could vary between $1: 2-1$ : 3 . From our data for the Petran variety $\left(P_{1}\right)$, the only sample for which the ratio between chlorophyll types $a /$ $b$ of chlorophyll was 1:2 was the methanol extract (sample 1). The ratio in the other three extracts was 1:3. For both the methanol and ethanol extracts of the Plamen $\left(\mathrm{P}_{2}\right)$ variety, we found a Chl $a: \mathrm{Chl} b$ ratio of $1: 4$. The variations in the relative contents of chlorophylls $a$ and $b$ may be related to disturbances at the level of PS II [40]. Wavelength scans of all extracts of C. officinalis

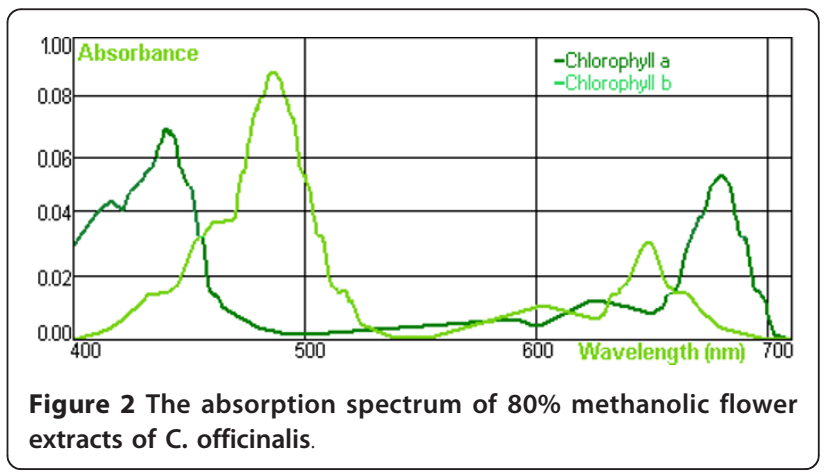




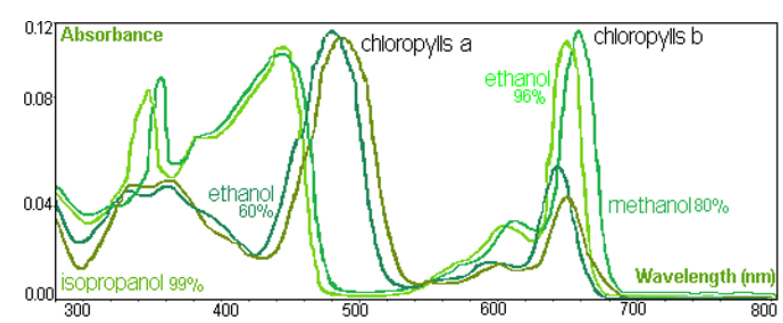

Figure 3 Absorption spectra of C. officinalis flower in methanol $80 \%$, ethanol $96 \%$, isopropanol $99 \%$ and ethanol $60 \%$.

are shown in Figure 3. Specific absorption patterns enabled us to identify particular molecule groups and domains. One of the most important functions of carotenoids is the photoprotection of the photosynthetic apparatus by quenching triplet chlorophyll, singlet oxygen and other reactive species.

Carotenoids are structural components of light-harvesting chlorophyll $a / b$ protein complexes of PSII (LHCII) and PS I. They are involved in both the stabilization of LHCII trimers and the assembly of LHCII monomers [41]. To be useful as a measure of cellular antioxidant potential, absorbance at the selected wavelength should be mainly influenced by the DPPH radical and not by interferences caused by antioxidants, such as carotenoids and polyunsaturated fatty acids. Given that signals at wavelengths below $530 \mathrm{~nm}$ are strongly influenced by carotenoids, this could result in misinterpretation of the measured absorbance decrease when a wavelength is chosen that is influenced by both the $\mathrm{DPPH}$ radical and the absorptive properties of the extract, as occurs for extracts containing carotenoids. To a large extent, this kind of interference can be eliminated by measuring the absorbance of a reference sample containing the same extract as the sample containing DPPH.

Our efforts to quantify the total content of carotenoids in extracts reveal the importance of the solvent selected for sample extraction. Methanol extracted carotenoid pigments more effectively than any of the other solvents tested.

\section{Discussion}

We used two spectrophotometric methods, which involve the capture of free radicals in solution by DPPH and estimation of the total antioxidant potential (FRAP), to provide complementary sources of evidence for the different antioxidant potentials of extracts from $C$. officinalis flowers. By correlating the composition of phenolic compounds with antioxidant action in flower tissues, we found that extracts from the C. officinalis variety Plamen $\left(\mathrm{P}_{2}\right)$ had higher antioxidant potential than extracts from the Petran $\left(\mathrm{P}_{1}\right)$ variety. The relationship between antioxidant activity and flavonoid content was not linear, which suggests that the antioxidant activity depends on the total polyphenol content. Volatile organic bio compounds are directly amenable to analysis by gas chromatography, a technique of unsurpassed separation capacity.

Flavonoids are considered favoured bio compounds as chemotaxonomic markers in plants because they show large structural diversity and are chemically stable.

The methodology developed in this work, identification and quantification of major phenolic compounds by samples used more easily accessible FC methods for determination of polyphenols.

Method based on the classical norms and on the standard operating procedures developed in the Biochemistry Department, gave for each validation criterion a value in agreement with the limitations of the previously cited norms.

\section{Conclusions}

Antioxidant components in the extracts were identified by combined HPLC methods and spectrophotometric analysis. Through spectrophotometric analysis of the extracts in the UV-VIS range, we identified specific phenolic acids and flavonoids.

Methanol enabled more efficient extraction of flavonoids than the other solvents tested. The highest antioxidant activity correlated to the polyphenol content was obtained for extracts prepared using methanol. Biochemical analysis determined the content of assimilator pigments, as well as flavonoids, which were expressed as equivalents of $\mathrm{QE}$. After the spectrophotometric dosage of flavonoids expressed in $\mathrm{QE}$ was obtained, the values included the following: 96.17/34.8 QEmg/100 mL.

According to the European Pharmacopeia, the minimal condition of quality was of $0.4 \%$ total flavonoids expressed in QE. The raw material quality of C. officinalis (Flores Calendulae) marigolds was conditioned by the major content in flavons and polyphenols, which accumulated during the flowering period.

\section{Methods}

\section{Plant Samples}

Plants were collected from a field site at the Teaching Base of USAMVB Timisoara. The two types of plant specimens, the variety Petran $\left(\mathrm{P}_{1}\right)$ and Plamen $\left(\mathrm{P}_{2}\right)$ were collected and tagged on the spot using standard techniques (the varieties were grown under the same conditions).

The plant specimens that were preserved as herbarium specimens were identified using the standard reference for this purpose [42], compared with specimens from the Department of Plant Resources, and identified with the help of taxonomists from the Department of Plant Phytotechny at the USAMVB Timisoara. 


\section{Extraction of Active Biologic Compounds}

The four solvents used for extraction were methanol (80\%), ethanol (96\%), isopropanol (100\%) and ethanol (60\%). The general process of maceration on a small scale involved placing suitably crushed plant material, or a moderately coarse powder made from it, in a closed vessel and adding the selected solvent to allow the solvent sufficient time to penetrate the cell wall to solubilize the constituents within the cells and for the resulting solution to penetrate the cell wall outwards. As the system is static, except for the occasional shaking, the process of extraction works by molecular diffusion, which is very slow. A closed vessel is used to prevent evaporation of the solvent during the extraction period, and thus avoids batch-to-batch variation.

At the end of the maceration process, when equilibrium has been reached, the solution is filtered by passage through a special press.

The concentrations of active constituents in the strained and expressed liquids, sometimes called miscella, are identical, and can thus be combined. Given that the expressed liquid may be cloudy with colloidal and small particles, a certain amount of time is often necessary for coagulation and settling of particulate matter.

The settled matter is filtered using a filter press or any other suitable apparatus.

The maceration extraction method is the simplest non-selective method. C. officinalis flowers were kept in a cold environment in contact with the solvent at room temperature $\left(17-22^{\circ} \mathrm{C}\right)$ for $14 \mathrm{~h}$, stirring occasionally before the extract was separated. An additional round(s) of maceration increased the efficiency of the process. The extracted product was first mixed with $1 / 2-2 / 3$ of the total quantity of solvent, after which the liquid was separated and the residue released.

It comes in contact with the rest of the solvent, resulting in one ninth quantity of solution mining. The two liquids extract and filter together after another 24 hours.

Owing to the relatively reduced thermal stability of natural aromatic compounds, the process was conducted in the presence of the antioxidant ascorbic acid $(0.5 \% \mathrm{w} /$ v) [43]. Extraction rate (1: $10 \mathrm{~g}$ of flowers powder $/ \mathrm{mL}$ solvent).

\section{High Performance Liquid Chromatography (HPLC)}

Polyphenol-containing extracts of C. officinalis flowers were separated on a $C_{18}(150 \mathrm{~mm} \times 4.6 \mathrm{~mm}, 3 \mu \mathrm{m})$ column, with a mobile phase consisting of three different solvents, using an Agilent 1100 HPLC apparatus. The flow rate was $0.7 \mathrm{~mL} / \mathrm{min}$ and the injection volume was $20 \mu \mathrm{L}$. For the first separation system (ternary solvent), which was used for the separation of flavanols and phenolic acids, the initial mobile phase consisted of $2 \%$ acetic acid in water/methanol. For separation of flavonols the second system (binary solvent: $0.25 \mathrm{mM}$ phosphate buffer, $\mathrm{pH}=2.5$ /acetonitrile), polyphenolic compounds were separated using a third system (binary solvent: $0.1 \%$ formic acid in water/methanol). The column temperature was adjusted at $20^{\circ} \mathrm{C}$.

Phenolic compounds were detected at $260 \mathrm{~nm}$. The separated compounds were identified by comparing their retention time (Rt) and UV spectra with those of authentic standards. Compounds were quantified using a standard calibration curve.

Total phenolic and flavonoid contents were quantified as the sum of the related identified phenolic compounds. The compounds were identified by comparing with standards of each identified compound using Rt, the absorbance spectrum profile, and also by running the samples after the addition of pure standards [35].

\section{Total polyphenol content}

The total polyphenol content was calculated using the FC reagent and $\mathrm{Na}_{2} \mathrm{CO}_{3} 10 \%$; ulterior the maximum absorption was evaluated by the obtained coloration, in relation to the calibration curve. The total content of phenolic compounds was evaluated using colorimetry based on the chemical reduction of the mixture of tungsten and molybdenum oxides to form a blue product that absorbs strongly at $760 \mathrm{~nm}$.

The formation of blue compounds derived from FCreactive phenols was independent of the structures of the phenolic compounds, enabling quantification of the full complement of phenolics from plant extracts. Approximately $10 \mathrm{~g}$ of pulverized plant material was extracted with $100 \mathrm{~mL}$ solvent. The total concentration of phenolic compounds in the extract was calculated through comparison with a curve prepared in a similar way based on measurement made using known concentrations of GA ranging between $0-00 \mu \mathrm{g} \mathrm{GA} / 100 \mathrm{~mL}$. The total content of phenols in samples was expressed in equivalents of GA $(\mathrm{mg})$ in a gram of dried plant material.

Levels of polyphenols were expressed in $\mathrm{mg} / \mathrm{g}$ equivalents of GA in relation to a final concentration $20 \mu \mathrm{g} /$ $\mathrm{mL}$.

\section{Total flavonoid content}

The combined level of all flavonoids was calculated by reaction with a $3 \%(\mathrm{w} / \mathrm{v})$ solution of $\mathrm{AlCl}_{3}$ (anhydrous salt) prepared in ethanol.

Concentrations were correlated with absorption values by using a calibration curve constructed using known concentrations of QE. The total content in flavonoids expressed in $\mathrm{mg} / \mathrm{g}$ (QE equivalents) was related to a final concentration by $20 \mu \mathrm{g} / \mathrm{mL}$. 


\section{Estimation of antioxidant activity using DPPH}

Approximately $10 \mathrm{~g}$ of pulverized plant material was extracted in $100 \mathrm{~mL}$ of an $80 \%$ aqueous solution of ethanol (or other solvents) at the room temperature for $1 \mathrm{~h}$.

The extracts were filtered, and the filters were left to evaporate in a dry environment.

The percentage free radical scavenging activity was determined using the formula (1), where Ai was the absorption before the tested extract adding and Af was the absorption value after a 5 min reaction time:

$$
100 \cdot(\mathrm{Ai}-\mathrm{Af}) / \mathrm{Ai}
$$

The neutralization effect of the $\mathrm{DPPH} \circ$ free radical was calculated at three different concentrations of methanol and ethanol extracts: portions of $0.05 \mathrm{~mL}$ from extracts of 10,5 and $2.5 \mathrm{mg} / \mathrm{mL}$ were mixed with $2.95 \mathrm{~mL}$ solution $\mathrm{DPPH} \circ$ in the cuvette used for spectrophotometry.

After a 5-min reaction time, the absorption at $420 \mathrm{~nm}$ was compared with that of methanol. QE was used as a positive control. At a concentration of $2.5 \mathrm{mM}$, QE was able to fully neutralize (the level of $\mathrm{DPPH}^{\circ}$ radical used).

\section{Ferric Reducing Antioxidant Power (FRAP) assays}

We used a simple spectrophotometric method to evaluate the antioxidant power of the plant extracts. This involved reduction of ferric tripyridyltriazine [Fe(III)TPTZ] to coloured ferrous tripyridyltriazine [Fe(II)TPTZ] at low $\mathrm{pH}$.

The coloured product absorbs strongly at $593 \mathrm{~nm}$.

\section{Pigment Analysis}

All samples were extracted in acetone. The pigments were extracted from C. officinalis flower with $80 \%$ acetone. We used the specific absorption coefficients of chlorophylls $a$ and $b$, and carotenoids (Car) to estimate their levels using spectrophotometry. All of the chlorophylls contained two main absorption bands of radiations: one on the blue side or near the UV portion of the electromagnetic spectrum, and the other on the red side or near the IR region of the electromagnetic spectrum. The lack of a significant absorption on the green side of the spectrum confers on chlorophylls their characteristic green or blue-greenish colors.

This method determines the amount $(\mathrm{mg})$ in $100 \mathrm{~mL}$ of carotenoid extract. The pigment compounds calculated according to Equations 2, 3 and 4:

$$
\begin{aligned}
& \text { Chl } a(\mu \mathrm{g} / \mathrm{g} \text { dry matter })=\left(15.65 \mathrm{~A}_{666}-7.34 \mathrm{~A}_{653}\right) \\
& \text { Chl } b(\mu \mathrm{g} / \mathrm{g} \text { dry matter })=\left(27.05 \mathrm{~A}_{653}-11.21 \mathrm{~A}_{666}\right)
\end{aligned}
$$

$$
\mathrm{x}=\mathrm{Ay} /\left(A_{1 \mathrm{~cm}}^{1 \%} \times 100\right)
$$

The working methods were based on the Ro Pharmacopeia 1993 techniques in conformity with international standards (Ph. Eur. Reference Standards and WHO, ICRS, 2010, [44-47].

\section{Reagents and Other Materials}

$\mathrm{QE}$ and GA were obtained from Sigma-Aldrich (St. Louis, MO, USA), whereas all other reagents were obtained from Merck. All chemicals and reagents were of analytical grade.

\section{Statistical Analysis}

All the results of spectrophotometric determinations of flavones and polyphenols were reproduced after establishing the confidence interval calculated for an average of a minimum of five determinations. The statistical differences between the means were determined using a two-tailed paired Student's t-test.

\section{Additional material}

Additional file 1: Table 1- Relative levels of compounds from $C$. officinalis extracts that have antioxidant potential.

Additional file 2: Table 2 -Spectrophotometrically determined carotenoids of $C$. officinalis flower in different solvents.

\begin{abstract}
Abbreviations
DPPH: 2, 2-diphenyl-1-picrylhydrazyl; FRAP: ferric reducing power; UV-VIS: ultraviolet-visible; HPLC: high-performance liquid chromatography; QE: quercetin; GA: gallic acid; FC: Folin-Ciocalteu; [Fe(III)-TPTZ]: ferric tripyridyltriazine; [Fe(II)-TPTZ]: ferrous tripyridyltriazine; Car: carotenoids; Chl al Chl $b$ : chlorophyllsa and $b$.
\end{abstract}

\section{Acknowledgements}

This paper was published during the project "Postdoctoral School of Agriculture and Veterinary Medicine", POSDRU/89/1.5/S/62371, co-financed by the European Social Fund through the Sectorial Operational Programme for the Human Resources Development 2007-2013.

\section{Author details}

${ }^{1}$ Chemistry and Biochemistry Department,Banat's University of Agricultural Sciences and Veterinary Medicine from Timisoara, Calea Aradului, no. 119, Timisoara, 300645 Romania. ²Department of Phytotechny, Banat's University Banat's University of Agricultural Sciences and Veterinary Medicine from Timisoara, Calea Aradului, no. 119, Timisoara, 300645 Romania.

\section{Authors' contributions}

$\mathrm{MB}$ conceived the study, participated in the design and co-ordination of the experiments and data interpretation and helped draft the manuscript. CZC produced samples, performed data analysis and data interpretation. All authors read and approved the final manuscript.

\section{Competing interests}

The authors declare that they have no competing interests.

Received: 1 April 2012 Accepted: 27 April 2012 Published: 27 April 2012 


\section{References}

1. Basch E, Bent S, Foppa I, Haskmi S, Kroll D, Mele M, Szapary P, Ulbricht C, Vora M, Yong S: Marigold (Calendula officinalis L.): an evidence-based systematic review by the Natural Standard Research Collaboration. J Herb Pharmacother 2006, 6:135-59.

2. Calendula officinalis-L. (HTML). Plants For A Future (PFAF) [http://www.pfaf. org/user/Plant.aspx?LatinName=Calendula+officinalis], Retrieved 2011/11/04.

3. Interactive Flora of NW Europe: Calendula officinalis, Retrieved 2011/08/12.

4. National Institutes of Health: "Calendula" (HTML). Herbs and Supplements. U.S. National Library of Medicine; Retrieved 2011/10/19.

5. Muley BP, Khadabadi SS, Banarase NB: Phytochemical Constituents and Pharmacological Activities of Calendula officinalis Linn (Asteraceae). Trop J Pharm Res 2009, 8:455-465.

6. Tanaka T, Matsuo Y, Kouno I: Review: Chemistry of Secondary Polyphenols Produced during Processing of Tea and Selected Foods. Int J Mol Sci 2010, 11:14-4.

7. Chakraborthy GS: Phytochemical screening of Calendula Officinalis Linn leaf extract by TLC. Int J Res Ayurveda Pharma 2010, 1:131-134.

8. Neukiron H, D'Ambrosio M, Dovia J, Guerriero A: Simultaneous Quantitative Determination of Eight Triterpenoid Monoesters from Flowers of 10 Varieties of Calendula officinalis L. and Characterisation of A New Triterpenoid Monoester. Phytochem Anal 2004, 15:30-35.

9. Giuliano G, Tavazza R, Diretto G, Beyer P, Taylor MA: Metabolic engineering of carotenoid biosynthesis in plants. Trends Biotechnol 2008, 26:139-145.

10. Kishimoto S, Maoka T, Sumitomo K, Ohmiya A: Analysis of carotenoid composition in petals of calendula (Calendula officinalis L.). Biosci Biotechnol Biochem 2005, 69:2122-8.

11. Preethi KC, Kuttan R: Wound healing activity of flower extract of Calendula officinalis. J Basic Clin Physiol Pharmacol 2009, 20:73-9.

12. Preethi KC, Kuttan G, Kuttan R: Antioxidant potential of Calendula officinalis Flowers in vitro and in vivo. Pharmaceutical Biol 2006, 44:691-697.

13. Guinot P, Gargadennec A, Valette G, Fruchier A, Andary C: Primary flavonoids in marigold dye: extraction, structure and involvement in the dyeing process. Phytochem Anal 2008, 19:46-51.

14. Braga PC, Dal Sasso M, Culici M, Spallino A, Falchi M, Bertelli A, Morelli R, Lo Scalzo R: Antioxidant activity of Calendula officinalis extract: inhibitory effects on chemiluminescence of human neutrophil bursts and electron paramagnetic resonance spectroscopy. Pharmacology 2009, 83:348-55.

15. Mezzomo N, Martínez J, Ferreira SRS: Economic viability of SFE from peach almond, spearmint and marigold. J Food Eng 2011, 103:473-479.

16. Hadfield RA, Vlahovic TC, Khan T: The use of marigold therapy for podiatric skin conditions. Foot Ankle J 2008, 1:1-8.

17. Muley BP, Khadabadi SS, Banarase NB: Phytochemical Constituents and Pharmacological Activities of Calendula officinalis Linn (Asteraceae). Tropical J Pharm Res 2009, 8:455-465.

18. Silva EJR, Gonçalves ES, Aguiar F, Evêncio LB, Lyra MMA, Coelho MCOC, Fraga MCCA, Wanderley AG: Toxicological studies on hydroalcohol extract of Calendula officinalis L. Phytotherapy Research 2007, 21:332-336.

19. Naguib NY, Khalil MY, El Sherbeny SE: A comparative study on the productivity and chemical constituents of various sources and species of Calendula plants as affected by two foliar fertilizers. J App/ Sci Res 2005, 1:176-189

20. Khalid KA, Teixeira da Silva JA: Yield, essential oil and pigment content of Calendula officinalis L. flower heads cultivated under salt stress conditions. Sci Hort 2010, 126:297-305.

21. Paolini J, Barboni T, Desjobert JM, Djabou N, Muselli A, Costa J: Chemical composition, intraspecies variation and seasonal variation in essential oils of Calendula arvensis L. Biochem Syst Ecol 2010, 38:865-874.

22. Fronza M, Heinzmann B, Hamburger M, Laufer S, Merfort I: Determination of the wound healing effect of Calendula extracts using the scratch assay with 3T3 fibroblasts. J Ethnopharmacol 2009, 126:463-467.

23. Bezbradica D, Milic-Aškrabic J, Petrovic SD, Šiler-Marinkovic S: An investigation of influence of solvent on the degradation kinetics of carotenoids in oil extracts of C. officinalis. J Serb Chem Soc 2005, 70:115-124.

24. Lu S, Li L: Carotenoid metabolism: biosynthesis, regulation, and beyond. J Integrative Plant Biol 2008, 50:778-785.

25. Fonseca YM, Catini CD, Vicentini FTMC, Nomizo A, Gerlach RF, Fonseca MJV: Protective effect of Calendula officinalis extract against UVB-induced oxidative stress in skin: Evaluation of reduced glutathione levels and matrix metalloproteinase secretion. J Ethnopharmacol 2010, 127:596-601.

26. Iriani $R$, Maldonade $D$, Rodriguez-Amaya $B$, Adilma $R$, Scamparini P: Carotenoids of yeasts isolated from the Brazilian ecosystem. Food Chem 2008, 107:145-150.

27. Raal A, Kirsipuu K: Total flavonoid content in varieties of Calendula officinalis L. originating from different countries and cultivated in Estonia. Nat Prod Res 2011, 25:658-62.

28. Preethi K, Chandran RK: Effect of Calendula officinalis Flower Extract on Acute Phase Proteins, Antioxidant Defense Mechanism and Granuloma Formation During Thermal Burns. J Clin Biochem Nutr 2008, 43:58-64.

29. Faria RL, Cardoso LM, Akisue G, Pereira CA, Junqueira JC, Jorge AO, Santos Júnior PV: Antimicrobial activity of Calendula officinalis, Camellia sinensis and chlorhexidine against the adherence of microorganisms to sutures after extraction of unerupted third molars. J Appl Oral Sci 2011, 19:476-82.

30. Butnariu M, Palicica RD: Pharmaceutical ointment based on marigold flowers grown in zinc-enriched soils and process for preparing the same. In Buletinul Oficial de Proprietate Industriala, Patent Applications. Volume 3. OSIM, RO122836-B1; 2010:1-18.

31. Preethi KC, Siveen KS, Kuttan R, Kuttan G: Inhibition of metastasis of B16F10 melanoma cells in C57BL/6 mice by an extract of Calendula officinalis L flowers. Asian Pac J Cancer Prev 2010, 11:1773-9.

32. Luo J, Zhang L, Wan M, He S, Yang Y: Determination of quercetin and kaempferol in Dysosma plants by RP-HPLC. Zhongguo Zhong Yao Za Zhi 2010, 35:3021-3023.

33. Danielski L, Campos LMAS, Bresciani LFV, Hense H, Yunes RA, Ferreira SRS: Marigold (Calendula officinalis L.) oleoresin: Solubility in $\mathrm{SC}-\mathrm{CO}_{2}$ and composition profile. Chem Eng Proc 2007, 46:99-106.

34. Raal A, Kirsipuu K, Must R, Tenno S: Content of total carotenoids in Calendula officinalis L. from different countries cultivated in Estonia. Nat Prod Commun 2009, 4:35-8.

35. Gazim Z, Rezende C, Fraga S, Dias Filho B, Nakamura C, Cortez D: Analysis of the essential oils from Calendula officinalis growing in Brazil using three different extraction procedures. Brazil J Pharm Sci 2008, 44:391-395.

36. Silva EJ, Costa-Silva JH, Evêncio LB, Fraga Mdo C, Coelho MC, Wanderley AG: Reproductive assessment of hydroalcohol extract of Calendula officinalis L. in Wistar rats. Phytother Res 2009, 23:1392-8.

37. Leach MJ: C. officinalis and wound healing: A systematic review. Wounds 2008, 20:236-243.

38. Okoh OO, Sadimenko AA, Afolayan AJ: The Effects of Age on the Yield and Composition of the Essential Oils of Calendula officinalis. J Appl Sci 2007, 7:3806-3810

39. Liu Z, Yan H, Wang K, Kuang T, Zhang J, Gui L, An X, Chang W: Crystal structure of spinach major light-harvesting complex at $2.72 \AA$ resolutions. Nature 2004, 428:287-292.

40. Guskov A, Kern J, Gabdulkhakov A, Broser M, Zouni A, Saenger W: Cyanobacterial photosystem II at 2.9- $\AA$ resolution and the role of quinones, lipids, channels and chloride. Nat Struct Mol Biol 2009, 16:334-342.

41. Ballottaria M, Girardona J, Dall'Ostoa L, Bassi R: Evolution and functional properties of Photosystem II light harvesting complexes in eukaryotes. Biochim Biophys Acta Bioenerg 2012, 1817:143-157.

42. European Pharmacopoeia. PHARMEUROPA 2011, 23: [http://www.edqm eu/en/News-and-General-Information-43.html], Retrieved 2011/10/15.

43. Granger KL, Gallagher RS, Fuerst EP, Alldredge JR: Comparison of seed phenolic extraction and assay methods. Methods Ecol Evol 2011, 2:691-698

44. Flora European. In Paperback Set Edited by: Tutin TG, Heywood VH, Burges NA, Valentine DH, Walters SM, Webb DA 2010, 5:135.

45. Ph. Eur. Reference Standards. [http://www.edgm.eu/en/Ph-Eur-ReferenceStandards-627.html], Retrieved 2011/10/16

46. WHO: International Chemical Reference Substances. (ICRS) 2010 [http:// www.edgm.eu/en/WHO-International-Chemical-Reference-Substances-ICRS1393.html], Retrieved 2011/10/18.

47. ISO-14502-1:2005; and AOAC 941.15.AOAC, 2003.

doi:10.1186/1752-153X-6-35

Cite this article as: Butnariu and Coradini: Evaluation of Biologically Active Compounds from Calendula officinalis Flowers using Spectrophotometry. Chemistry Central Journal 2012 6:35 